PROCEEDINGS OF THE

AMERICAN MATHEMATICAL SOCIETY

Volume 127, Number 10, Pages 3043-3048

S 0002-9939(99)04977-1

Article electronically published on April 23, 1999

\title{
EXAMPLES OF VECTOR BUNDLES ADMITTING UNIQUE ASD CONNECTIONS ON QUATERNION-KÄHLER MANIFOLDS
}

\author{
YASUYUKI NAGATOMO \\ (Communicated by Ronald A. Fintushel)
}

\begin{abstract}
We prove a series of rigidity theorems. Examples of higher rank ASD vector bundles are given on quaternion-Kähler manifolds, which admit the one and only ASD connections modulo the gauge equivalence.
\end{abstract}

\section{INTRODUCTION}

In the present paper, we are interested in anti-self-dual (ASD) connections on complex vector bundles over positive quaternion-Kähler manifolds. By definition, a quaternion-Kähler manifold is a $4 n$-dimensional Riemannian manifold for which the linear holonomy group can be reduced to $S p(1) \cdot S p(n)$. A positive quaternionKähler manifold means a compact quaternion-Kähler manifold with a positive scalar curvature. (This definition is followed by LeBrun and Salamon [4].) Anti-selfduality can be defined over quaternion-Kähler manifolds in the same way as in the 4-dimensional case (for example, Mamone Capria and Salamon [5]). For brevity, a vector bundle with an ASD connection is called an ASD bundle.

We will prove a series of rigidity theorems for ASD connections. As an easy consequence of Salamon [10] and LeBrun-Salamon [4], we obtain the classification of ASD connections on complex line bundles over positive quaternion-Kähler manifolds (see $\S 4$ Appendix). Lemma 4.1 asserts that the moduli space of ASD connections on a line bundle is one point as a set. However, in the case of higher dimensional quaternion-Kähler manifolds, there exists a vector bundle of rank $>1$ which admits only one ASD connection up to the gauge equivalence.

Theorem 1.1. Let $\left(E_{p}, \nabla\right)$ be an irreducible ASD bundle of rank $r$ with a unitary structure over $G r_{2}\left(\mathbb{C}^{n+2}\right)$, where $p$ is an integer. If the first Chern class of $E$ is $(r p+1)(x-y)$ and the second Chern class is $\frac{p}{2}(r-1)(r p+2)(x-y)^{2}+\left(x^{2}-x y+y^{2}\right)$, then $E_{p}$ is a homogeneous bundle and the rank $r$ equals to $n$. The ASD connection $\nabla$ is gauge equivalent to the canonical connection. (The cohomology classes $x$ and $y$ are defined in $\S 2$.)

Remark 1.2. If the dual bundle $E_{p}^{*}$ is also taken into account $\left(c_{1}\left(E_{p}^{*}\right)=\right.$ $(r p+1)(-x+y))$, we obtain the same conclusion.

Received by the editors October 15, 1997 and, in revised form, December 17, 1997.

1991 Mathematics Subject Classification. Primary 53C07.

(C)1999 American Mathematical Society 
To prove Theorem 1.1 in the case $p=0$, we employ the theory of monads. (For a full detail of the theory of monads, we recommend the book [9].) The theory of monads usually has the advantage of providing a deformation of holomorphic structures. In our case, we make use of monads to show that there exists only one holomorphic structure on the given bundle.

Using this rigidity theorem $(p=0)$ and Lemma 4.1, we make a proof of Theorem 1.1 complete. A calculation of the Chern classes reduces the general cases to the first one.

If we take the Whitney sum of a homogeneous line bundle of degree $-x+y$ and $E_{0}$ on $G r_{2}\left(\mathbb{C}^{n+2}\right)$, the solution space to the ASD equation modulo the gauge equivalence on the direct sum of vector bundles is no longer one point and is identified with an open cone over $P\left(\mathbb{C}^{n+2}\right)$ [8]. Theorem 1.1 and Lemma 4.1 assure that the vertex of the moduli space is really one point and represents the canonical connection.

\section{Preliminaries}

Let $M$ be a quaternion-Kähler manifold and $E$ be a complex vector bundle on $M$. A connection on $E$ is called an anti-self-dual (ASD) connection if its curvature 2 -form is invariant under the $S p(1)$ action of the quaternion structure. (See, for example, [5].)

A quaternion-Kähler manifold $M$ has the Salamon twistor space $Z$ [10]. If $M$ is a positive quaternion-Kähler manifold, the twistor space is a Fano manifold which is a projective algebraic manifold and has the positive first Chern class [10]. An ASD bundle pulls back to a holomorphic vector bundle on $Z$ with the induced connection (for example, [5]).

In the present paper, we need the theory of monads on the twistor space $F^{2 n+1}$ of 2-plane complex Grassmannian $G r_{2}\left(\mathbb{C}^{n+2}\right)$. Hence we review the topology of the generalized flag manifold $F^{2 n+1}$ :

$$
F^{2 n+1}=\frac{S U(n+2)}{S(U(1) \times U(n) \times U(1))} .
$$

In other words, $F^{2 n+1}$ is represented as follows:

$$
F^{2 n+1}=\left\{(l, V) \mid 0 \in l \subset V \subset \mathbb{C}^{n+2}, \operatorname{dim} l=1 \text { and } \operatorname{dim} V=n+1\right\} .
$$

Then the twistor fibration $\pi: F \rightarrow G r_{2}\left(\mathbb{C}^{n+2}\right)$ is

$$
\pi((l, V))=l \oplus V^{\perp} .
$$

So, note that this is not a holomorphic fibration [10]. (When no confusion can arise, the dimension $2 n+1$ will be omitted.) Now, we describe the ring structure of the cohomology of $F$. The twistor space $F^{2 n+1}$ has double holomorphic fibrations to $\mathbb{P}^{n+1}$ and $\mathbb{P}^{n+1 *}$ such that

$$
p_{1}:(l, V) \longrightarrow[l] \text { and } p_{2}:(l, V) \longrightarrow[V] .
$$

We denote by $x$ and $y$ pull-back elements of $H^{2}\left(F^{2 n+1}, \mathbb{Q}\right)$ of the standard positive generators of $H^{2}\left(\mathbb{P}^{n+1}, \mathbb{Z}\right)$ and $H^{2}\left(\mathbb{P}^{n+1 *}, \mathbb{Z}\right)$ respectively. Then, by the result of [1], the cohomology ring $H^{*}\left(F^{2 n+1}, \mathbb{Q}\right)$ is generated by $x$ and $y$. More precisely, it is isomorphic to the quotient ring by an ideal generated by $x^{n+2}, y^{n+2}$ 
and $x^{n+1}-x^{n} y+\cdots+(-1)^{n+1} y^{n+1}$. On the other hand, using the twistor fibration, $H^{*}\left(G r_{2}\left(\mathbb{C}^{n+2}\right), \mathbb{Q}\right)$ is regarded as a subring of $H^{*}(F, \mathbb{Q})[1]$. Consequently, an element of $H^{*}\left(G r_{2}\left(\mathbb{C}^{n+2}\right), \mathbb{Q}\right)$ may be written with $x$ and $y$.

Since $F$ is a Fano manifold, the Picard group is isomorphic to $H^{2}\left(F^{2 n+1}, \mathbb{Z}\right)$. Hence, we denote by $\mathcal{O}(p, q)$ the line bundle on $F$ for which the first Chern class is $p x+q y$, where $p, q \in \mathbb{Z}$.

\section{Rigidity THEOREMS}

Throughout this section, we do not distinguish between an ASD bundle $E_{p}$ on $G r_{2}\left(\mathbb{C}^{n+2}\right)$ and its pull-back on the twistor space $F^{2 n+1}$, and we use the same symbol $E_{p}$ for both.

Proposition 3.1. Let $E_{0}$ be a vector bundle of rank $r$ as in Theorem 1.1. Then the pull-back bundle $E_{0}$ on $F$ is a cohomology bundle of the monad

$$
\mathcal{O}(-1,0) \stackrel{\alpha}{\longrightarrow} \underline{-} \stackrel{\beta}{\longrightarrow} \mathcal{O}(0,1),
$$

where $\underline{V}$ is a trivial bundle $F \times V$ of rank $r+2$.

Proof. Our proof is done in the same way as in a proof of Main Theorem 2 in [8]. More precisely, we use only induction in the dimension of the base manifold and some vanishing theorems. In the case of $n=1\left(\mathbb{C} P^{2}\right)$, Buchdahl [2] shows our result. (Note that $c_{2}\left(E_{0}\right)=0$ in this case.)

By induction, vanishing theorems [8] imply that $H^{1}\left(F^{2 n+1}, E_{0}(0,-1)\right)$ and $H^{1}\left(F^{2 n+1}, E_{0}^{*}(-1,0)\right)$ are one-dimensional vector spaces. Using the identities of $\operatorname{End}\left(H^{1}\left(F^{2 n+1}, E_{0}(0,-1)\right)\right)$ and $\operatorname{End}\left(H^{1}\left(F^{2 n+1}, E_{0}^{*}(-1,0)\right)\right)$, we define a monad

$$
W_{2}(-1,0) \longrightarrow \underline{V} \longrightarrow W_{1}^{*}(0,1),
$$

where $W_{1}=H^{1}\left(F^{2 n+1}, E_{0}(0,-1)\right)^{*}$ and $W_{2}=H^{1}\left(F^{2 n+1}, E_{0}^{*}(-1,0)\right)^{*}$. The similar method in proofs of Proposition 4.6 and Theorem 4.7 in [8] yields that $\underline{V}$ is a trivial vector bundle of rank $r+2$.

To describe homomorphisms in the monad (3.1), we make use of the expression of $F^{2 n+1}$ as a homogeneous space. For brevity, $S U(n+2)$ is denoted by $G$ and $S(U(1) \times U(n) \times U(1))$ is denoted by $K_{Z}$. Let $\mathbb{C}^{n+2}$ be the standard representation space of $G$ with a $G$-invariant hermitian inner product $h$. Now we denote by $e$ (resp. $f$ ) the highest (resp. lowest) weight vector with the norm 1 in $\mathbb{C}^{n+2}$. Then, by the restriction of the action of $G$ to $K_{Z}$, we have two irreducible representation spaces $\mathbb{C} e$ and $\mathbb{C} f$ of $K_{Z}$. Under this notation, we have

$$
\mathcal{O}(-1,0)=G \times_{K_{Z}} \mathbb{C} e, \text { and } \mathcal{O}(0,1)=G \times_{K_{Z}} \mathbb{C} f .
$$

Hence, for example, an element of $\mathcal{O}(-1,0)$ is denoted by $[g, c e]$, where $c$ is a complex number and $[g, c e]$ is the coset represented by $(g, c e) \in G \times \mathbb{C} e$.

We can prove the following proposition in the same way as in proofs of Proposition 5.1 and Lemmas 5.2 and 5.4 in [8].

Proposition 3.2. Let $\alpha$ and $\beta$ be homomorphisms in the monad (3.1). Then, there exist $A \in \operatorname{Hom}\left(\mathbb{C}^{n+2}, V\right)$ and $B \in \operatorname{Hom}\left(V, \mathbb{C}^{n+2}\right)$ such that

$$
\alpha([g, c e])=([g], c A g e), \quad \beta([g], v)=[g, h(B v, g f) f],
$$

where $g \in S U(n+2), c \in \mathbb{C}$, and $v \in V$. Moreover, $A$ is injective, $B$ is surjective and the composition $B \circ A$ is the identity of $\mathbb{C}^{n+2}$ up to non-zero constant multiple. 
Corollary 3.3. The rank $r$ of $E_{0}$ is greater than or equal to $n$.

Proof. From Proposition 3.2, the dimension of $V$ must be greater than or equal to $n+2$.

Theorem 3.4. Let $E_{0}$ be a vector bundle satisfying the conditions in Theorem 1.1. If $E_{0}$ has an irreducible $A S D$ connection, then the rank of $E_{0}$ is equal to $n$ and we have an identification between $V$ and $\mathbb{C}^{n+2}$.

Proof. First, we assume that $H^{0}\left(E_{0}\right) \neq 0$. In general, a holomorphic section $s \in$ $H^{0}(Z, E)$ corresponds to a covariant constant section of $E_{0}$ over $M$, where $Z$ is the twistor space of a quaternion-Kähler manifold $M$ and $E$ is an ASD bundle on $M$. (See, for example, $[11$, p. 422] or [6]. A direct computation in [6] shows this fact.) This implies that $E_{0}$ has a trivial subbundle with a flat connection. This is a contradiction with the irreducibility and so, $H^{0}\left(E_{0}\right)=0$.

The first row of the display of the monad (3.1) and the Bott-Borel-Weil theorem yield that $H^{i}(\operatorname{Ker} \beta) \cong H^{i}\left(E_{0}\right)$ for $i=0, \cdots, 2 n+1$. Consequently, $H^{0}(\operatorname{Ker} \beta)=$ 0 . By the first column of the display of the monad (3.1), we obtain

$$
0 \longrightarrow V \longrightarrow H^{0}(\mathcal{O}(0,1)) \longrightarrow H^{1}(\operatorname{Ker} \beta) \longrightarrow 0 .
$$

Using again the Bott-Borel-Weil theorem, we have $H^{0}(\mathcal{O}(0,1)) \cong \mathbb{C}^{n+2}$ as the representation space of $G$. Hence, $\operatorname{dim} H^{1}\left(E_{0}\right)=\operatorname{dim} H^{1}(\operatorname{Ker} \beta)=n+2-\operatorname{dim} V$ and so, $\operatorname{dim} V \leqq n+2$. Since $\operatorname{rank} E_{0}=\operatorname{dim} V-2$, we obtain $\operatorname{rank} E_{0} \leqq n$. However, Corollary 3.3 asserts that rank $E_{0} \geqq n$. Consequently we have rank $E_{0}=$ $n$ and $H^{1}\left(E_{0}\right)=0$. Then, (3.2) yields that $V \cong H^{0}(\mathcal{O}(0,1)) \cong \mathbb{C}^{n+2}$.

Hence we assume that the rank of $E_{0}$ is $n$ and $V$ in the monad (3.1) is the standard representation space $\mathbb{C}^{n+2}$ of $S U(n+2)$.

It is easy to see that isomorphic monads induce isomorphic cohomology bundles. Now we focus our attention on the isomorphic classes of monads (3.1).

Theorem 3.5. For any choice of homomorphisms $\alpha$ and $\beta$ in the monad (3.1), we have the isomorphic cohomology bundle.

Proof. We assume that monads (MI) and $\left(\mathrm{MI}^{\prime}\right)$ are isomorphic to each other; in other words, the following diagram is commutative:

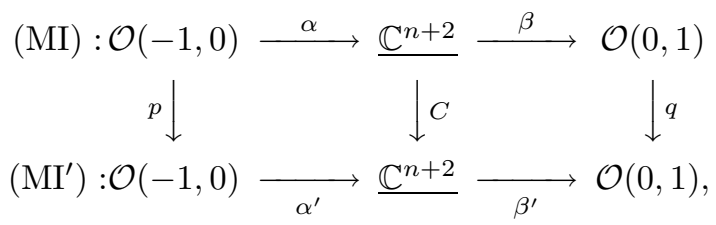

where $C$ is an automorphism of $\underline{\mathbb{C}^{n+2}}, p$ and $q$ are automorphisms of $\mathcal{O}(-1,0)$ and $\mathcal{O}(0,1)$, respectively. The Bott-Borel-Weil theorem implies that $H^{0}(\operatorname{End}(\mathcal{O}(-1,0))$ $\cong H^{0}\left(\operatorname{End}(\mathcal{O}(0,1)) \cong \mathbb{C}\right.$; we also have the identification between $H^{0}\left(\operatorname{End}\left(\underline{\mathbb{C}^{n+2}}\right)\right)$ and End $\left(\mathbb{C}^{n+2}\right)$. Consequently $p$ and $q$ can be regarded as non-zero constants and the automorphism $C$ is identified with an element in $A u t\left(\mathbb{C}^{n+2}\right)$. Then the commutative diagram and Proposition 3.2 yield that

$$
([g], C A g e)=p\left([g], A^{\prime} g e\right) \quad \text { and } \quad q h(B v, g f)=h\left(B^{\prime} C v, g f\right)
$$

for arbitrary $g \in G$ and $v \in \mathbb{C}^{n+2}$. From the irreducibility of the standard representation of $G$, we have $C A=p A^{\prime}$ and $q B=B^{\prime} C$. 
However, using again Proposition 3.2, there exist non-zero constants $c$ and $c^{\prime}$ such that $B A=c I d$ and $B^{\prime} A^{\prime}=c^{\prime} I d$, where $I d$ is the identity of $\mathbb{C}^{n+2}$. Consequently, if we put $C=c A^{\prime} A^{-1}, p=c$ and $q=c^{\prime}$, we obtain the desired isomorphism.

Proof of Theorem 1.1. Under the identification in Proposition 3.2, we can regard homomorphisms $\alpha$ and $\beta$ in the monad (3.1) as the identities of $\mathbb{C}^{n+2}$ from Theorem 3.5. This is the standard monad induced by the standard representation of $S U(n+2)$. (This terminology is defined in [7, Definition 4.4].) Hence the cohomology bundle is identified with a homogeneous bundle $G \times{ }_{K_{Z}} \mathbb{C}^{n}$ with the holomorphic structure induced by the canonical connection, where $\mathbb{C}^{n}$ is the orthogonal complement of $\mathbb{C} e \oplus \mathbb{C} f$ in $\mathbb{C}^{n+2}$. Then Theorem 3.4 in [7] implies the desired result.

Finally we take account of the case that $p \neq 0$. An easy computation shows that

$$
c_{1}\left(E_{p}(-p, p)\right)=x-y, \quad c_{2}\left(E_{p}(-p, p)\right)=x^{2}-x y+y^{2} .
$$

The proof of Lemma 4.1 in $\S 4$ and Theorem 3.4 in [7] yield that the line bundle $\mathcal{O}(-p, p)$ is nothing but the pull-back of a homogeneous ASD line bundles over $G r_{2}\left(\mathbb{C}^{n+2}\right)$. Consequently, $E_{p}(-p, p)$ is also the pull-back of an ASD bundle. Our theorem in the case $p=0$ implies that $E_{p}(-p, p)$ is isomorphic to the direct sum of $E_{0}$ and a trivial vector bundle as a holomorphic vector bundle and so, under the hypothesis of irreducibility, we obtain $E_{p} \cong E_{0}(p,-p)$. Hence $E_{p}$ is a homogeneous vector bundle of rank $n$ and the holomorphic structure of $E_{p}$ is induced by the canonical connection. We may use again Theorem 3.4 in [7].

\section{Appendix}

In this section, we give the classification of ASD connections on complex line bundles over positive quaternion-Kähler manifolds. Now $M$ is a $4 n$-dimensional positive quaternion-Kähler manifold.

Lemma 4.1. Let $(L, \nabla)$ be an ASD complex line bundle with a hermitian metric over a positive quaternion-Kähler manifold $M$. Then $L$ is a trivial line bundle and $\nabla$ is a trivial connection, unless $M=G r_{2}\left(\mathbb{C}^{n+2}\right)$. If $M$ is a complex Grassmannian $G r_{2}\left(\mathbb{C}^{n+2}\right)$, then $L$ is a homogeneous line bundle and $\nabla$ is gauge equivalent to the canonical connection.

Proof. A positive quaternion-Kähler manifold $M$ is simply connected from Salamon [10]. Let $M$ not be a complex Grassmannian $G r_{2}\left(\mathbb{C}^{n+2}\right)$. Then LeBrun and Salamon [4] showed that the second homotopy group $\pi_{2}(M)$ is finite. Consequently, the second cohomology group $H^{2}(M, \mathbb{Z})$ vanishes and so $c_{1}(L)=0$. In the case of a line bundle $L$, the Yang-Mills functional is bounded from below by $c \int_{M} c_{1}(L)^{2} \wedge \Omega^{n-1}$ $[5,3]$, where $c$ is a positive constant and $\Omega$ is the fundamental 4 -form on $M$. In particular, ASD connections minimize the Yang-Mills functional and attain the minima $c \int_{M} c_{1}(L)^{2} \wedge \Omega^{n-1}[5,3]$. Hence the connection $\nabla$ is flat. Using again the fact that $\pi_{1}(M)=0$, we obtain the desired result.

If $M$ is $G r_{2}\left(\mathbb{C}^{n+2}\right)$, then we take account of the pull-back bundle on the twistor space $F$. The pull-back bundle is a holomorphic line bundle (for example [5]), which is denoted by $\tilde{L}$. Since $F$ is Fano, the Kodaira vanishing theorem implies that the holomorphic structure of a line bundle is uniquely determined by its first Chern class. On the other hand, the standard theory of a compact simply-connected homogeneous Kähler manifold shows that for every integral cohomology class $\alpha$ on $F$, there exists a homogeneous line bundle of which the first Chern class is $\alpha$ and 
the canonical connection induces the holomorphic structure on this line bundle. Therefore $\tilde{L}$ is a homogeneous line bundle with the canonical connection up to the gauge equivalence. Then Theorem 3.4 in [7] yields that $L$ is also a homogeneous bundle and $\nabla$ is gauge equivalent to the canonical connection.

\section{REFERENCES}

[1] I.N.Bernstein, I.M.Gelfand and S.I.Gelfand, Schubert cells and the cohomology of the spaces $G / P$, L.M.S.Lecture Notes 69 (1982), Cambridge University Press, 115-140

[2] N.P.Buchdahl, Instantons on $\mathbb{C} P^{2}$, J. Differential Geom. 24 (1986), 19-52 MR 88b:32066

[3] K.Galicki and Y.S.Poon, Duality and Yang-Mills fields on quaternionic Kähler manifolds, J. Math. Phys. 32 (1991), 1263-1268 MR 92i:53024

[4] C.LeBrun and S.M.Salamon, Strong rigidity of positive quaternion-Kähler manifolds, Invent. Math. 118 (1994), 109-132 MR 95k:53059

[5] M.Mamone Capria and S.M.Salamon, Yang-Mills fields on quaternionic spaces, Nonlinearity 1 (1988), 517-530 MR 89k:58064

[6] Y.Nagatomo, Vanishing theorem for cohomology groups of $c_{2}$-self-dual bundles on quaternionic Kähler Manifolds, Differential Geom. Appl. 5 (1995), 79-97 MR 95m:32041

[7] Y.Nagatomo, Representation theory and ADHM-construction on quaternionic symmetric spaces, a preprint

[8] Y.Nagatomo, Another type of instanton bundles on $G_{2}\left(\mathbb{C}^{n+2}\right)$, to appear in Tokyo J. Math.

[9] C.Okonek, M.Schneider and H.Spindler, "Vector bundles on complex projective spaces", Progress in Math. 3, Birkhäuser, Boston, (1980) MR 81b:14001

[10] S.M.Salamon, Quaternionic Kähler Manifolds, Invent. Math. 67 (1982), 143-171 MR 83k:53054

[11] R.S.Ward and R.O.Wells Jr, "Twistor Geometry and Field Theory", Cambridge monographs on Math. Physics, Cambridge University Press (1989) MR 91b:32034

Institute of Mathematics, University of Tsukuba, Tsukuba-Shi, Ibaraki 305-8571, JAPAN

E-mail address: nagatomo@math.tsukuba.ac.jp 